\title{
Expansion and Lack Thereof in Randomly Perturbed Graphs
}

\author{
Abraham D. Flaxman
}

Abstract. This paper studies the expansion properties of randomly perturbed graphs. These graphs are formed by adding, for example, a random 1-out or very sparse ErdősRényi graph to an arbitrary connected graph.

The central results show that there exists a constant $\delta$ such that when any connected $n$-vertex base graph $G_{0}$ is perturbed by adding a random 1-out then, with high probability, the resulting graph has $e(S, \bar{S}) \geq \delta|S|$ for all $S \subseteq V$ with $|S| \leq \frac{3}{4} n$. When $G_{0}$ is perturbed by adding a random Erdös-Rényi graph, $\mathbb{G}_{n, \epsilon / n}$, the expansion of the perturbed graph depends on the structure of the base graph. A necessary condition for the base graph is given under which the resulting graph is an expander with high probability.

The proof techniques are also applied to study rapid mixing in the small-worlds graphs described by Watts and Strogatz in [Watts and Strogatz 98] and by Kleinberg in [Kleinberg 00]. Analysis of Kleinberg's model shows that the graph stops being an expander exactly at the point where a decentralized algorithm is effective in constructing a short path.

The proofs of expansion rely on a method of summing over subsets of vertices that allows an argument based on the first-moment method to succeed.

\section{Introduction}

Developing models of complex networks has been a major focus in the fields of physics, mathematics, and computer science. Empirical studies of many large networks gleaned from the real world have revealed that, unlike the classical

(C) A K Peters, Ltd.

|542-795I/07 \$0.50 per page 
models of Erdős-Rényi random graphs developed for applications to probabilistic combinatorics, many of the complex networks that surround us today have high clustering coefficients and power-law degree sequences. This observation has driven the development of numerous alternate distributions for random graphs, which often are described by some generative procedure.

Unfortunately, it is much easier to propose a generative procedure than to refute one, which has led to the preponderance of models available today. The copious models for real-world graphs may not withstand the test of time any better than the Erdős-Rényi distribution, however. This motivates the approach pursued in the present paper. Instead of studying a particular model for generating graphs with the hopes of finding it "more realistic" than previously proposed models, this paper considers an approach for incorporating randomness into network modeling that is less model-specific.

In this paper, a complex network is viewed as being composed of a base graph and a random perturbation. The general goal in this framework is to show that some property is likely to hold for a wide variety of base graphs under a very gentle random perturbation. For example, [Flaxman and Frieze 04] shows that if a network is generated from any connected base graph with $n$ vertices, perturbed by taking the symmetric difference with $\epsilon n$ random edges, then, whp, ${ }^{1}$ if the network is connected then it will have diameter $\mathcal{O}\left(\epsilon^{-1} \log n\right)$.

This directly extends Bollobás and Chung's pioneering study of a cycle plus a random matching [Bollobás and Chung 88] and can be viewed as work along the lines of "How many random edges make a dense graph Hamiltonian?" and subsequent studies on the effects of adding a few random edges to dense graphs [Bohman et al. 03, Bohman et al. 04, Krivelevich et al. 06]. It is also similar in spirit to the smoothed analysis of algorithms, introduced by Spielman and Teng [Spielman and Teng 01], which has been used to explain why algorithms perform better in practice than worst-case bounds predict (see also [Spielman and Teng 03, Banderier et al. 03, Becchetti et al. 03, Beier and Vöcking 04, Deshpande and Spielman 05]). Also similar are the hybrid graphs studied in [Chung and Lu 04, Andersen et al. 04], which explicitly model long and short edges.

In addition to the perturbation models like those considered on sparse random instances in [Flaxman and Frieze 04], this paper will consider non-uniform perturbations, in the spirit of Jon Kleinberg's small-world model [Kleinberg 00] and long-range percolation in finite graphs studied in [Benjamini and Berger 01, Coppersmith et al. 02, Biskup 04], and also the graph that both these models build upon, the small-world model of Watts and Strogatz [Watts and Strogatz 98].

\footnotetext{
${ }^{1}$ In this paper, with high probability (whp) means that a sequence of events $\left\{\mathcal{E}_{n}\right\}$ has $\operatorname{Pr}\left[\mathcal{E}_{n}\right] \rightarrow 1$ as $n \rightarrow \infty$.
} 


\begin{tabular}{|c|c|}
\hline Perturbation & Expander whp? \\
\hline 1 -out & Yes, for any connected $G_{0}$ \\
$\mathbb{G}_{n, \epsilon / n}$ & Not if $G_{0}$ has a bad partition \\
Watts-Strogatz Small World & Not if $G_{0}$ has a bad partition \\
Kleinberg Small World & Yes, for any connected $G_{0}$, if $r<r_{\max }\left(G_{0}\right)$ \\
\hline
\end{tabular}

Table I. Conditions for expansion under several perturbations.

\section{I.I. Results and Applications}

The main technical development in this paper is a technique for understanding when randomly perturbed graphs exhibit expansion properties. This is motivated by the success of expansion bounds on more traditional random instances. For sufficiently dense Erdős-Rényi graphs, the first-moment method provides a simple way to obtain whp a lower-bound on expansion. This paper provides a new method of accounting that permits a similar first-moment-method approach to be used on randomly perturbed graphs.

For clear presentation, this new application of the first-moment method is presented in the proof of an expansion property for a random graph $G$ formed by perturbing any connected graph $G_{0}$ by adding a random 1-out (which is the random graph formed by adding an edge from each vertex to another vertex chosen uniformly at random and ignoring the directions of the edges).

Theorem I.I. For any sufficiently small $\delta>0$, for any $n$-vertex connected graph $G_{0}$, and for $R \sim \mathbb{G}_{n, 1-\text { out }}$, the perturbed graph $G=G_{0}+R$ has the following property whp: for all $S \subset V$ with $|S| \leq \frac{3}{4} n$, at least $\delta|S|$ edges connect $S$ and $\bar{S}$.

This technology is also applied to similar random graphs, to yield results summarized in Table 1.

\subsection{History of Expansion in Random Graphs}

A close connection between edge expansion, vertex expansion, spectral gap, and mixing time has emerged since 1970 [Cheeger 70, Alon and Milman 85, Alon 86, Sinclair and Jerrum 89, Lawler and Sokal 88, Wu 05, Chung 05]. Through this link, many different results on random graphs can be related to expansion properties. In regular and nearly regular random graphs, bounds on the secondlargest eigenvalue of the adjacency matrix give bounds on expansion [Füredi and Komlós 81, Juhász 81, Friedman et al. 89, Alon 97, Friedman 03, Feige and Ofek 05]. In a graph with a power-law degree distribution or other non-regular graphs, the eigenvalues of the adjacency matrix are not necessarily related to eigenvalues 
of the Laplacian and expansion. Both have been investigated theoretically and experimentally in recent years [Faloutsos et al. 99, Monasson 99, Benjamini and Berger 01, Mihail and Papadimitriou 02, Cooper and Frieze 03, Chung et al. 03, Mihail et al. 03, Flaxman et al. 05].

In the empirical study of complex networks occurring in the real world, examining Laplacian eigenvalues has revealed that some real networks are expanders and others are not [Blandford et al. 03, Estrada 06]. This has led to the development of web graph models that specifically avoid being good expanders [Flaxman et al. 04].

Algorithmically, there are many benefits to knowing that a graph is an expander (for example, rapid mixing, disjoint paths and routing, and robustness to attacks), and there are many other benefits to knowing that a graph is not an expander (for example, high-quality cuts, divide-and-conquer algorithms, and compressing data). Expansion may be less universal to real-world graphs than other properties observed empirically like local clustering and power-law degree distributions.

\section{I.3. Notation}

Undirected edges are sets of two vertices, and edge $\{u, v\}$ will be abbreviated as $u v$ when it is not confusing to do so. For any graph $H$, let $E(H)$ denote the edge set of $H$, and let $V(H)$ denote the vertex set of $H$. For sets $S, T \subseteq V(H)$, let $e_{H}(S, T)$ denote the number of edges between $S$ and $T$ in $H$, and let $e_{H}(S)$ denote the number of edges in the graph induced by vertex set $S$ (the induced graph is denoted $H[S])$. Let $\operatorname{deg}_{H}(v)$ denote the degree of $v$ in $H$. The subscript $G$ of $e_{G}(S, T), e_{G}(S)$, and $\operatorname{deg}_{G}(v)$ will be omitted when referring the graph $G$ if it is not confusing to do so.

The "blackboard" notation $R \sim G_{n, p}$ means that $R$ is a random graph distributed according to $G_{n, p}$.

\section{I.4. Distributions for Random Graphs}

Perturbed graph I $\left(\mathcal{P}_{\mathbf{1}}\right)$. The randomly perturbed graph that appears in Theorem 1.1 is a random graph generated by starting with base graph $G_{0}$ and adding a random 1-out $\left(\mathbb{G}_{n, 1-\text { out }}\right.$ is the distribution of random graphs where every vertex chooses a neighbor uniformly at random and adds an edge to it.) The random graph $G=G_{0}+R$ where $R \sim \mathbb{G}_{n, 1 \text {-out }}$ is studied primarily to illustrate the central technique of this paper, although it is a reasonably small perturbation. On average, it changes the degree of every vertex by 2 . 
Perturbed graph $2\left(\mathcal{P}_{2}\right)$. In the context of studying the effects of randomness on complex networks without making drastic assumptions about the distribution of the randomness, it would be better to use a perturbation that does not change the base graph as much as a 1-out does. This can be accomplished by starting with base graph $G_{0}$ and adding a sparse Erdős-Rényi random graph $\left(\mathbb{G}_{n, \epsilon / n}\right.$ is the distribution of random graphs, where each of the $\left(\begin{array}{l}n \\ 2\end{array}\right)$ candidate edges appears independently with probability $\epsilon / n$.) The random graph $G=G_{0}+R$ where $R \sim \mathbb{G}_{n, \epsilon / n}$ is studied in [Flaxman and Frieze 04], which shows that, whp, $\operatorname{diam}(G)=\mathcal{O}\left(\epsilon^{-1} \log n\right)$. Since, on average, this perturbation changes the degree of every vertex by only $\epsilon$, the local effects of the perturbation are quite minimal.

Small-world graph I $\left(\mathcal{S} \mathcal{W}_{1}\right)$. The small-world model of Watts and Strogatz is generated by starting with a base graph $G_{0}$ and an ordering of the edges $E\left(G_{0}\right)$. (In [Watts and Strogatz 98], $G_{0}$ is a ring of $n$ vertices with each vertex connected to its $k$ nearest neighbors with $k \gg \ln n$, and the edges are ordered in a particular way that is implicit in the description of the perturbation.) The base graph is perturbed in the following fashion: proceed through the edges according to the ordering and, for each edge, with probability $p$, randomly rewire this edge to a vertex chosen uniformly at random, with duplicate edges forbidden; otherwise leave the edge in place.

Small-world graph $2\left(\mathcal{S W}_{2}\right)$. Kleinberg's small-world graph is a random digraph generated by starting with a base graph $G_{0}$ and a distance function $d(\cdot, \cdot)$ on the vertices of $V\left(G_{0}\right)$. (In [Kleinberg 00], $G_{0}$ is primarily taken to be an $n \times n$ grid, where $V=[n]^{2}$, and $u v$ is an edge if $d_{1}(u, v) \leq p$; the distance function is taken to be the $\ell_{1}$ norm.) The base graph is perturbed by adding $q$ random edges from every vertex independently at random, where the $i$ th edge from vertex $v$ is denoted by $e_{v, i}$ and is chosen according to the distribution

$$
\operatorname{Pr}\left[e_{v, i}=v w\right]=d(v, w)^{-r} /\left(\sum_{u \neq v} d(v, u)^{-r}\right)
$$

for all $w \neq v$ (here, $r$ is a parameter of the model).

Comparison of $\mathcal{S} \mathcal{W}_{1}$ and $\mathcal{S} \mathcal{W}_{2}$. $\mathcal{S} \mathcal{W}_{2}$ is often viewed as a generalization of $\mathcal{S} \mathcal{W}_{1}$. The big difference is that, while $\mathcal{S} \mathcal{W}_{1}$ rewires edges uniformly at random, $\mathcal{S} \mathcal{W}_{2}$ includes the parameter $r$, which controls the degree to which the underlying network is willing to form long-distance connections. There is also a subtle difference between these two models. While $\mathcal{S} \mathcal{W}_{1}$ randomly rewires each edge of the underlying graph with probability $p$ (which, for a $d$-regular graph, results in $d p$ random edges expected from each vertex), $\mathcal{S W}_{2}$ adds $q$ random edges from each vertex. This sounds very similar to $q=d p$, and it is similar, but it 
is also different, in a very important way. Graphs from the $\mathcal{S} \mathcal{W}_{2}$ distribution are expanders whp, while graphs from the $\mathcal{S} \mathcal{W}_{1}$ distribution are not necessarily so.

\subsection{Outline of What Follows}

Section 2 proceeds with the proof of Theorem 1.1, which uses a new method of First-moment-method accounting to show that $G=G_{0}+R$ has $e(S, \bar{S}) \geq \delta|S|$ for all $S$ whp when $R$ is a 1-out $\left(\mathcal{P}_{1}\right)$.

Section 3 considers a more gentle perturbation, where $R$ is distributed as $\mathbb{G}_{n, \epsilon / n}$ instead of as a 1-out. In this case, $G$ is not necessarily an expander, and a criterion for $G_{0}$ of having a "bad partition" is shown to prevent $G$ from satisfying the expansion property whp. The same results are also shown to hold for Watts-Strogatz random graphs $\left(\mathcal{S} \mathcal{W}_{1}\right)$. In particular, when $G_{0}$ is a cycle with edges connecting each vertex to its $k$ nearest neighbors, or when $G_{0}$ is a $d$-dimensional grid, it contains a bad partition and hence the perturbed graph is not an expander whp.

Section 4 considers the $\mathcal{S} \mathcal{W}_{2}$ perturbation, where $G_{0}$ is perturbed by a nonuniform $q$-out, in which each random edge from $v$ chooses a vertex $w$ with probability related to distance from $v$ to $w$ under some distance function $d(\cdot, \cdot)$, according to $\operatorname{Pr}\left[e_{v, i}=v u\right]=d(v, u)^{-r} / \sum_{w \neq v} d(v, w)^{-r}$. For $q=1$ and $r=0$, this reduces to the base-graph-plus-1-out considered in Section 2, and the techniques from that section are shown to extend to $r>0$ for grid-like graphs. These techniques show that when $G_{0}$ is a $d$-dimensional grid, $G$ is an expander for any $r<d$, and, furthermore, there is a threshold at $r=d$, at which point $G$ is no longer an expander.

This shows that the transition from expanding to non-expanding occurs precisely at the point where a local algorithm can find polylogarithmic-length paths in the network.

\section{Perturbing Any Connected $G_{0}$ with a 1-out Yields Expander}

The proof of Theorem 1.1 is an application of the first-moment method, and relies on a moderately precise calculation of the expected number of sets $S$ that violate the bound $e(S, \bar{S}) \leq \delta|S|$. This is achieved by considering the sets with $|S| \leq \gamma n$ and $|S|>\gamma n$ separately for an appropriately chosen constant $\gamma$.

Proof of Theorem I.I. A straightforward way to obtain an upper bound on the probability that there exists a set $S \subseteq V$ with $|S| \leq \frac{3}{4} n$ and $e(S, \bar{S}) \leq \delta|S|$ is the 
following: let $Z_{S}$ be an indicator random variable for the event that a particular set $S$ satisfies these conditions, and calculate an upper bound on the expected value of the sum $Z=\sum_{S \subseteq V} Z_{S}$. Showing that the expected value tends to 0 as $n$ tends to $\infty$ yields a bound that proves the theorem, because, for any nonnegative random variable, $\operatorname{Pr}[Z \geq 1] \leq \mathrm{E}[Z]$ (this deceptively simple inequality is honored with the title "first-moment method"; see, for example, [Alon and Spencer 00]).

Unlike the simple application of the first-moment method, which is sufficient to show that $G \sim \mathbb{G}_{n, k \text {-out }}$ is likely to be an expander when $k$ is a great enough constant, to make this calculation yield results about a perturbed graph will require considering the structure of the set $S$ in the base graph $G_{0}$.

The key trick is to use a special tour of $G_{0}$ to describe each set $S$; let $T$ be a spanning tree of $G_{0}$, and let $W=\left(e_{1}, e_{2}, \ldots, e_{2(n-1)}\right)$ be an Euler tour of the multigraph formed by including every edge of $T$ twice. This is to say that $W$ is a sequence of edges that gives a circuit in $G$ that traverses each edge of $T$ exactly two times. Such a tour exists because doubling every edge of $T$ makes the degree of every vertex even. For any set $S$, let $\mathbf{I}_{S} \in\{0,1\}^{2(n-1)}$ be the incidence vector with $\mathbf{I}_{S}(i)=1$ if and only if $v_{i} \in S$, where $v_{i}$ is the $i$ th vertex of walk $W$. Let $e\left(\mathbf{I}_{S}\right)=\left|\left\{i: \mathbf{I}_{S}(i) \neq \mathbf{I}_{S}(i+1)\right\}\right|$ denote the number of times the Euler tour crosses the boundary of $S$. There is a direct relationship between $e_{T}(S, \bar{S})$ and $e\left(\mathbf{I}_{S}\right)$. Since each edge of $T$ appears twice in $W$,

$$
e_{G_{0}}(S, \bar{S}) \geq e_{T}(S, \bar{S}) \geq e\left(\mathbf{I}_{S}\right) / 2 .
$$

To obtain a bound on the expected value of the sum $\sum_{S:|S|=s} Z_{S}$, let

$$
\mathcal{S}_{s, k}=\left\{S:|S|=s, e\left(\mathbf{I}_{S}\right)=k\right\}
$$

denote the collection of sets $S$ of size $s$ for which $W$ crosses the boundary of $S$ exactly $k$ times. Since every $S$ maps to a unique $\mathbf{I}_{S}$, it follows that

$$
\left|\mathcal{S}_{s, k}\right| \leq 2\left(\begin{array}{c}
2 n \\
k
\end{array}\right)
$$

because an incidence vector with $k$ changes in value can be described by giving the $k$ "change positions" and specifying whether the first bit is a 0 or a 1.

For $S \in \mathcal{S}_{s, k}$, equation (2.1) shows that $e_{G_{0}}(S, \bar{S}) \geq k / 2$, so, in order to have $e(S, \bar{S}) \leq \delta s$, it is necessary that $e_{G_{0}}(S, \bar{S}) \leq \delta s$ and $e_{R \backslash G_{0}}(S, \bar{S}) \leq \delta s-k / 2$. 
This implies that $e_{R}(S, \bar{S}) \leq 2 \delta s-k / 2$, which is impossible when $k>4 \delta s$. Thus,

$$
\begin{aligned}
\sum_{S:|S|=s} \mathrm{E}\left[Z_{S}\right] & \leq \sum_{k=1}^{4 \delta s} \sum_{S \in \mathcal{S}_{s, k}} \operatorname{Pr}\left[e_{R}(S, \bar{S}) \leq 2 \delta s\right] \\
& \leq \sum_{k=1}^{4 \delta s} 2\left(\begin{array}{c}
2 n \\
k
\end{array}\right) \operatorname{Pr}\left[e_{R}(S, \bar{S}) \leq 2 \delta s\right] \\
& \leq(8 \delta s)\left(\begin{array}{c}
2 n \\
4 \delta s
\end{array}\right) \operatorname{Pr}\left[e_{R}(S, \bar{S}) \leq 2 \delta s\right] \\
& \leq n\left(\frac{n e}{2 \delta s}\right)^{4 \delta s} \operatorname{Pr}\left[e_{R}(S, \bar{S}) \leq 2 \delta s\right], \quad \text { for } \delta \leq 1 / 8 .
\end{aligned}
$$

Finishing the calculation requires an upper bound on $\operatorname{Pr}\left[e_{R}(S, \bar{S}) \leq 2 \delta s\right]$, for which it is necessary to consider the large and small sets $S$ separately.

Large sets expand. When $s=|S| \geq \gamma n$, Chernoff's bound (see, for example, [Janson et al. 00]) gives a sufficient upper bound for the probability. Considering just the edges directed from $S$ shows that $\mathrm{E}\left[e_{R}(S, \bar{S})\right] \geq s\left(1-\frac{s}{n}\right)$, so Chernoff's bound gives

$$
\operatorname{Pr}\left[e_{R}(S, \bar{S}) \leq 2 \delta s\right] \leq \exp \left\{\frac{-s\left(1-\frac{s}{n}\right)\left(1-\frac{2 \delta}{1-s / n}\right)^{2}}{2}\right\} .
$$

So, for $\gamma n \leq s \leq \frac{3}{4} n$,

$$
\sum_{S:|S|=s} \mathrm{E}\left[Z_{S}\right] \leq n\left[\left(\frac{e}{2 \delta \gamma}\right)^{4 \delta} \exp \left\{-\frac{(1-8 \delta)^{2}}{8}\right\}\right]^{s} .
$$

For any constant $\gamma$, if $\delta$ is a sufficiently small constant then this upper bound is exponentially small in $n$.

Small sets expand. When $s=|S| \leq \gamma n$, a tighter bound on the probability can be obtained directly by

$$
\operatorname{Pr}\left[e_{R}(S, \bar{S}) \leq 2 \delta s\right] \leq\left(\begin{array}{c}
s \\
2 \delta s
\end{array}\right)\left(\frac{s}{n}\right)^{s-2 \delta s} \leq\left[\left(\frac{e}{2 \delta}\right)^{2 \delta}\left(\frac{s}{n}\right)^{1-2 \delta}\right]^{s}
$$

So

$$
\sum_{S:|S|=s} \mathrm{E}\left[Z_{S}\right] \leq n\left[\left(\frac{n e}{2 \delta s}\right)^{4 \delta}\left(\frac{e}{2 \delta}\right)^{2 \delta}\left(\frac{s}{n}\right)^{1-2 \delta}\right]^{s}=n\left[\left(\frac{e}{2 \delta}\right)^{6 \delta}\left(\frac{s}{n}\right)^{1-6 \delta}\right]^{s} .
$$

For $\delta$ sufficiently small and $\frac{3}{1-6 \delta} \leq s \leq \gamma n$, this upper bound is $o(1 / n)$. 
Tiny sets expand. For $\delta \leq \frac{1}{12}$, tiny sets $S$, of size $s \leq \frac{3}{1-6 \delta}$, will satisfy $e(S, \bar{S}) \geq \delta s$ because the base graph $G_{0}$ is connected, so $e(S, \bar{S}) \geq 1 \geq \delta \frac{3}{1-6 \delta}$.

Putting all this together shows that there exists $\delta>0$ such that

$$
\operatorname{Pr}\left[\text { exists } S:|S| \leq \frac{3}{4} n \text { and } e(S, \bar{S}) \leq \delta|S|\right] \leq \sum_{s=1}^{\frac{3}{4} n} \sum_{S:|S|=s} \mathrm{E}\left[Z_{S}\right]=o(1)
$$

\section{Gentler Perturbation Does Not Necessarily Yield Expander}

Adding a 1-out to a graph increases the average degree of a vertex by 2 . This is not much, but it is not nothing. This section investigates the effects of perturbing $G_{0}$ by adding a random instance of $\mathbb{G}_{n, \epsilon / n}$ (the Erdős-Rényi graph in which every candidate edge is included independently with probability $\epsilon / n)$. The intention of the parameterization $\epsilon / n$ is to indicate that $\epsilon$ should be thought of as a small constant, although the results of this section apply to any constant $\epsilon$.

An attempt to show that if $R \sim \mathbb{G}_{n, \epsilon / n}$ then the perturbed graph $G=G_{0}+R$ is an expander can begin by following in the footsteps of the proof of Theorem 1.1. Such a proof attempt will succeed in showing that whp the large sets in $G$ expand.

Theorem 3.I. For any $\epsilon>0$, for any sufficiently small $\delta>0$, for any $n$-vertex connected graph $G_{0}$, and for $R \sim \mathbb{G}_{n, \epsilon / n}$, the perturbed graph $G=G_{0}+R$ has the following property whp: for all $S \subseteq V$ with $e^{-\epsilon /(64 \delta)} n \leq|S| \leq \frac{3}{4} n$, at least $\delta|S|$ edges connect $S$ and $\bar{S}$.

Proof. Follow the proof of Theorem 1.1. The only new calculation this proof requires is a fresh application of Chernoff's bound. For this perturbation, $\mathrm{E}\left[e_{R}(S, \bar{S})\right]=\epsilon s\left(1-\frac{s}{n}\right)$, so

$$
\operatorname{Pr}\left[e_{R}(S, \bar{S}) \leq \delta s\right] \leq \exp \left\{-\frac{\epsilon s}{2}\left(1-\frac{s}{n}\right)\left(1-\frac{\delta}{\epsilon(1-s / n)}\right)^{2}\right\}
$$

Following the proof of Theorem 1.1 does not succeed in showing that small sets expand, however. Indeed, it should not show this, because it is not necessarily true. If $G_{0}$ has a bad partition (defined below) then whp $G$ is not an expander.

Definition 3.2. $G_{0}$ has a $\delta$-bad partition if and only if $V\left(G_{0}\right)$ can be partitioned into $k$ small sets $S_{1}, \ldots, S_{k}$ and a "left-over" set $\bar{S}$ for which the following inequalities 
hold:

$$
\begin{array}{ll}
\left|S_{i}\right| \leq \frac{1}{2} \epsilon^{-1} \ln n, & \text { for } i=1, \ldots, k \\
e_{G_{0}}\left(S_{i}, \bar{S}_{i}\right)<\delta\left|S_{i}\right|, & \text { for } i=1, \ldots, k \\
k=\omega\left(n^{1 / 2}\right) . &
\end{array}
$$

Theorem 3.3. For any $\epsilon>0$, any $G_{0}$, and $R \sim \mathbb{G}_{n, \epsilon / n}$, if $G_{0}$ has a $\delta$-bad partition, then whp there exists $i \in\{1, \ldots, k\}$ such that $e_{R}\left(S_{i}, \bar{S}_{i}\right)=0$, and hence $G=$ $G_{0}+R$ has e $\left(S_{i}, \bar{S}_{i}\right)<\delta\left|S_{i}\right|$.

Proof. The proof is a direct application of the second-moment method (as described in [Alon and Spencer 00]). Let $Z_{i}$ be an indicator random variable for the event $e_{R}\left(S_{i}, \bar{S}_{i}\right)=0$, and let $Z=\sum_{i=1}^{k} Z_{i}$. Then the probability that there exists $i \in\{1, \ldots, k\}$ such that $S_{i}$ fails to expand is $\operatorname{Pr}[Z=0] \geq \mathrm{E}[Z]^{2} / \mathrm{E}\left[Z^{2}\right]$.

Let $s_{i}=\left|S_{i}\right|$. Then

$$
\mathrm{E}[Z]=\sum_{i=1}^{k} \operatorname{Pr}\left[e_{R}\left(S_{i}, \bar{S}_{i}\right)=0\right]=\sum_{i=1}^{k}(1-\epsilon / n)^{s_{i}\left(n-s_{i}\right)}
$$

and

$$
\begin{aligned}
\mathrm{E}\left[Z^{2}\right] & =\sum_{i=1}^{k} \sum_{j=1}^{k} \operatorname{Pr}\left[e_{R}\left(S_{i}, \bar{S}_{i}\right)=0\right] \operatorname{Pr}\left[e_{R}\left(S_{j}, \bar{S}_{j}\right)=0 \mid e_{R}\left(S_{i}, \bar{S}_{i}\right)=0\right] \\
& =\sum_{i=1}^{k}(1-\epsilon / n)^{s_{i}\left(n-s_{i}\right)}\left(1+\sum_{j \neq i}(1-\epsilon / n)^{s_{j}\left(n-s_{i}-s_{j}\right)}\right) \\
& \leq \sum_{i=1}^{k}(1-\epsilon / n)^{s_{i}\left(n-s_{i}\right)}\left(1+(1+o(1)) \sum_{j=1}^{k}(1-\epsilon / n)^{s_{j}\left(n-s_{j}\right)}\right) \\
& \leq(1+o(1))\left(1+\sum_{i=1}^{k}(1-\epsilon / n)^{s_{i}\left(n-s_{i}\right)}\right)^{2} \\
& =(1+o(1))(1+\mathrm{E}[Z])^{2} .
\end{aligned}
$$

Since $\mathrm{E}[Z] \geq(1-o(1)) k e^{-\ln n / 2} \rightarrow \infty$,

$$
\operatorname{Pr}[Z=0] \geq(1-o(1)) \frac{\mathrm{E}[Z]^{2}}{(1+\mathrm{E}[Z])^{2}}=1-o(1) .
$$

This theorem shows that, for example, when $G_{0}$ is the $d$-dimensional grid graph, whp the perturbed graph is not an expander. 
Corollary 3.4. Let $G_{0}$ be a d-dimensional grid on $N=n^{d}$ vertices, and let $R \sim$ $\mathbb{G}_{N, \epsilon / N}$ for any $\epsilon>0$. Then, for any $\delta>0$, whp the graph $G=G_{0}+R$ has some $S \subseteq V$ with $|S|=\frac{1}{2} \epsilon^{-1} \ln N$ and $e(S, \bar{S})<\delta|S|$.

Proof. Partition $V\left(G_{0}\right)$ into subcubes, each containing $\ln N$ vertices. Each subcube $S_{i}$ has sides of length $(\ln N)^{1 / d}$, and, for any constant $\delta$ and $N$ sufficiently large,

$$
e_{G_{0}}\left(S_{i}, \bar{S}_{i}\right)=\mathcal{O}\left((\ln N)^{(d-1) / d}\right)<\delta \ln N
$$

On the other hand, if $G_{0}$ is a graph such that all small partitions satisfy the expansion condition, then Theorem 3.1 is sufficient to show that $G$ is an expander whp. For example, if $G_{0}$ consists of two expander graphs, each on $n / 2$ disjoint vertices, that are joined by a single edge, then $G$ will be an expander whp.

The proof of Theorem 3.3 goes through essentially without modification to show that the model studied by Watts and Strogatz $\left(\mathcal{S} \mathcal{W}_{1}\right.$ with $G_{0}$ a $k$-connected cycle) is not an expander for any $\delta$ if $k$ is any constant. On the other hand, when $k \gg \ln n$ (as in the original Watts-Strogatz specification), whp every vertex has at least one edge randomly rewired, so it follows from Theorem 1.1 that the resulting graph is an expander whp.

\section{Conditions for Expansion in Kleinberg's Small-World Graph}

In $\mathcal{S} \mathcal{W}_{2}$, when $r=0$ and $q=1$, this is exactly the case treated in Theorem 1.1. Making $q$ greater only increases the number of edges across any cut, so any connected base graph leads to an expander when $r=0$.

For $r>0$, the proof of expansion for sets of size at most $\beta n$ can proceed as in the proof of Theorem 1.1, provided that there is an upper bound on $\operatorname{Pr}\left[e_{v, i} \in S\right]$ of the form

$$
\text { for any } v \in V \text { and } S \subseteq V \text { with }|S|=s \leq \beta n, \operatorname{Pr}\left[e_{v, i} \in S\right] \leq C\left(\frac{s}{n}\right)^{\epsilon},
$$

where the constants $C>0$ and $\epsilon>0$ satisfy $C \beta^{\epsilon} \leq \frac{3}{4}$.

When the metric is the $\ell_{1}$ norm on the lattice $[n]^{d}$, such a bound exists for any $r<d$.

Theorem 4.I. Let $V=[n]^{d}$, and let $d(u, v)=\sum_{i=1}^{d}\left|u_{i}-v_{i}\right|$. Then, for any $r$ with $0 \leq r<d$, and for any $v \in V$ and $S \subseteq V$ with $|S|=s, \operatorname{Pr}\left[e_{v, i} \in S\right] \leq$ $C\left(\frac{s}{n}\right)^{d-r-1}$. 
Proof. For any $v \in V$, there are $\Theta\left(\ell^{d}\right)$ vertices with distance from $v$ of at most $\ell$, and there are $\Theta\left(\ell^{d-1}\right)$ vertices with distance from $v$ exactly $\ell$. For any $v \in V$ and $S \subseteq V$, let $s=|S|$, let $s_{\ell}$ be the number of vertices of $S$ that are at distance $\ell$ from $v$, and let $n_{\ell}$ be the number of vertices of $V$ that are at distance $\ell$ from $v$ :

$$
s_{\ell}=\left|\left\{w \in S: d_{1}(v, w)=\ell\right\}\right|, \quad n_{\ell}=\left|\left\{w \in V: d_{1}(v, w)=\ell\right\}\right| .
$$

Then

$$
\begin{aligned}
\operatorname{Pr}\left[e_{v, i} \in S\right] & =\frac{\sum_{w \in S} d_{1}(v, w)^{-r}}{\sum_{u \neq v} d_{1}(v, u)^{-r}} \\
& =\frac{\sum_{\ell=1}^{d n} s_{\ell} \ell^{-r}}{\sum_{\ell=1}^{d n} n_{\ell} \ell^{-r}} \\
& \leq \frac{\sum_{\ell=1}^{\Theta\left(s^{1 / d}\right)} \Theta\left(\ell^{d-1}\right) \ell^{-r}}{\sum_{\ell=1}^{\Theta\left(|V|^{1 / d}\right)} \Theta\left(\ell^{d-1}\right) \ell^{-r}} \\
& =\Theta\left(\frac{s^{(d-r) / d}}{|V|^{(d-r) / d}}\right) .
\end{aligned}
$$

For $d=2$, the upper bound on $r$ given in this bound is tight, and, when $r=d=2$, the resulting graph is not an expander.

Theorem 4.2. For $G_{0}$ an $n \times n \operatorname{grid}, d(\cdot, \cdot)=d_{1}(\cdot, \cdot)$, and $r \geq 2$, for any constant $\delta>0$, whp Kleinberg's small-world graph is not a $\delta$-expander.

Proof. To verify the theorem, consider the set $S=\{(x, y): x+y \leq k\}$, where $k=n / \ln n$, and calculate an upper bound on the expected number of random edges between $S$ and $\bar{S}$. This calculation can be simplified by considering sets $S_{\ell}=\left\{(x, y) \in V\left(G_{0}\right): x+y=\ell\right\}$. For any $i$ and $j$ with $i \leq k<j$,

$$
\begin{aligned}
\sum_{v \in S_{i}} \sum_{w \in S_{j}} d_{1}(v, w)^{-2} & \leq\left|S_{i}\right|\left((j-i+1) \frac{1}{(j-i)^{2}}+2 \sum_{\ell=1}^{\left|S_{j}\right|-(j-i)} \frac{1}{(j-i+2 \ell)^{2}}\right) \\
& \leq i\left(\frac{2}{j-i}+2 \sum_{\ell=1}^{i} \frac{1}{(j-i+2 \ell)^{2}}\right) \\
& \leq 2 i\left(\frac{2}{j-i}\right) .
\end{aligned}
$$


Also, for any $v \in V\left(G_{0}\right)$,

$$
\sum_{w \neq v} d_{1}(v, w)^{-2}=\Theta(\ln n) .
$$

Thus, an upper bound on the expected number of random edges between $S$ and $\bar{S}$ is given by the following (where $H_{j}=1+1 / 2+\ldots+1 / j$ denotes the $k$ th harmonic number):

$$
\begin{aligned}
\mathrm{E}\left[e_{R}(S, \bar{S})\right] & \leq \sum_{i=1}^{k} \sum_{j=k+1}^{n} \sum_{v \in S_{i}} \sum_{w \in S_{j}} q\left(\frac{d_{1}(v, w)^{-2}}{\sum_{u \neq v} d_{1}(v, u)^{-2}}+\frac{d_{1}(w, v)^{-2}}{\sum_{u \neq w} d_{1}(w, u)^{-2}}\right) \\
& \leq(2 q) \Theta\left((\ln n)^{-1}\right) \sum_{i=1}^{k} \sum_{j=k+1}^{n} 4 i\left(\frac{2}{j-i}\right) \\
& \leq(16 q k) \Theta\left((\ln n)^{-1}\right) \sum_{i=1}^{k}\left(H_{n}-H_{i}\right) \\
& =(16 q k) \Theta\left((\ln n)^{-1}\right)\left(k+k\left(H_{n}-H_{k}\right)\right) \\
& =\Theta\left(\frac{k^{2} \ln \ln n}{\ln n}\right) .
\end{aligned}
$$

Since $k=n / \ln n$ and $e_{G_{0}}(S, \bar{S})=\mathcal{O}(k)$, Markov's inequality shows that, for any constant $\delta$ with $\delta>0$, whp $e(S, \bar{S}) \leq \delta|S|$.

\section{Conclusion}

It is necessary to conclude that the expansion of a randomly perturbed graph depends on the base graph and the perturbation, and even seemingly similar perturbations can produce vastly different results. Although adding a random 1-out makes any connected graph an expander whp, such a simple statement is impossible for the gentler perturbation of adding a random $\mathbb{G}_{n, \epsilon / n}$. This is not a bad thing, however, as empirical observations show that among complex networks in the real world, some are expanders and others are not.

In the case of the small-world models of Watts and Strogatz and of Kleinberg, the difference in the distributions is quite subtle. Generally Kleinberg's model is viewed as a strict generalization of Watts and Strogatz's, but, in the context of expansion, the models are actually just different.

It is a pleasant surprise that Kleinberg's model stops being an expander exactly at the point where it becomes possible to find short paths with a decentralized 
algorithm. Perhaps the expansion threshold and existence of decentralized algorithms are fundamentally related in some way, but more likely not.

Acknowledgments. Thanks to Juan Vera, Haifeng Yu, and Phil Gibbons for helpful discussions.

\section{References}

[Alon 86] N. Alon. "Eigenvalues and Expanders." Combinatorica 6:2 (1986), 83-96.

[Alon 97] N. Alon and N. Kahale. "A Spectral Technique for Coloring Random 3Colorable Graphs." SIAM J. Comput. 26:6 (1997), 1733-1748.

[Alon and Milman 85] N. Alon and V. D. Milman. " $\lambda_{1}$, Isoperimetric Inequalities for Graphs, and Superconcentrators." J. Combin. Theory Ser. B 38:1 (1985), 73-88.

[Alon and Spencer 00] N. Alon and J. H. Spencer. The Probabilistic Method, Second edition, Wiley-Interscience Series in Discrete Mathematics and Optimization. New York: Wiley-Interscience, 2000.

[Andersen et al. 04] R. Andersen, F. Chung, and L. Lu. "Analyzing the Small World Phenomenon Using a Hybrid Model with Local Network Flow." In Algorithms and Models for the Web-Graph: Third International Workshop, WAW 2004, Rome, Italy, October 16, 2004, Proceeedings, Lecture Notes in Computer Science 3243, pp. 19-30. Berlin: Springer, 2004.

[Banderier et al. 03] C. Banderier, R. Beier, and K. Mehlhorn. "Smoothed Analysis of Three Combinatorial Problems." In Mathematical Foundations of Computer Science 2003: 28th International Symposium, MFCS 2003, Bratislava, Slovakia, August 25-29, 2003, Proceedings, Lecture Notes in Computer Science 2747, pp. 198207. Berlin: Springer, 2003.

[Becchetti et al. 03] L. Becchetti, S. Leonardi, A. Marchetti-Spaccamela, G. Schäfer, and T. Vredeveld. "Average Case and Smoothed Competitive Analysis of the Multi-Level Feedback Algorithm." In Proceedings of the 44th Symposium on Foundations of Computer Science (FOCS 2003), p. 462. Washington, DC: IEEE Computer Society, 2003.

[Beier and Vöcking 04] R. Beier and B. Vöcking. "Typical Properties of Winners and Losers in Discrete Optimization." In Proceedings of the 36th Annual ACM Symposium on Theory of Computing, Chicago, Illinois, USA, June 13-15, 2004, pp. 343352. New York: ACM Press, 2004.

[Benjamini and Berger 01] I. Benjamini and N. Berger. "The Diameter of Long-Range Percolation Clusters on Finite Cycles." Random Structures Algorithms 19:2 (2001), 102-111.

[Biskup 04] M. Biskup. "On the Scaling of the Chemical Distance in Long-Range Percolation Models." Ann. Probab. 32:4 (2004), 2938-2977.

[Blandford et al. 03] D. K. Blandford, G. E. Blelloch, and I. A. Kash. "Compact Representations of Separable Graphs." In Proceedings of the Fourteenth Annual ACM- 
SIAM Symposium on Discrete Algorithms, Baltimore, Maryland, January 12-14, 2003, pp. 679-688. Philadelphia, PA: SIAM, 2003.

[Bohman et al. 03] T. Bohman, A. Frieze, and R. Martin. "How Many Random Edges Make a Dense Graph Hamiltonian?" Random Structures Algorithms 22:1 (2003), $33-42$.

[Bohman et al. 04] T. Bohman, A. Frieze, M. Krivelevich, and R. Martin. "Adding Random Edges to Dense Graphs." Random Structures Algorithms 24:2 (2004), $105-117$.

[Bollobás and Chung 88] B. Bollobás and F. R. K. Chung. "The Diameter of a Cycle Plus a Random Matching." SIAM J. Discrete Math. 1:3 (1988), 328-333.

[Cheeger 70] J. Cheeger. "A Lower Bound for the Smallest Eigenvalue of the Laplacian." In Problems in Analysis: A Symposium in Honor of Salomon Bochner, edited by R. C. Gunning, pp. 195-199. Princeton, NJ: Princeton University Press, 1970.

[Chung 05] F. Chung. "Laplacians and the Cheeger Inequality for Directed Graphs." Ann. Comb. 9:1 (2005), 1-19.

[Chung and Lu 04] F. Chung and L. Lu. "The Small World Phenomenon in Hybrid Power Law Graphs." In Complex Networks, Lecture Notes in Physics 650, pp. 89104. Berlin: Springer, 2004.

[Chung et al. 03] F. Chung, L. Lu, and V. Vu. "Eigenvalues of Random Power Law Graphs." Ann. Comb. 7:1 (2003), 21-33.

[Cooper and Frieze 03] C. Cooper and A. Frieze. "Crawling on Simple Models of Web Graphs." Internet Math. 1:1 (2003), 57-90.

[Coppersmith et al. 02] D. Coppersmith, D. Gamarnik, and M. Sviridenko. "The Diameter of a Long-Range Percolation Graph." Random Structures Algorithms 21:1 (2002), 1-13.

[Deshpande and Spielman 05] A. Deshpande and D. A. Spielman. "Improved Smoothed Analysis of the Shadow Vertex Simplex Method." In Proceedings of the 46th Annual IEEE Symposium on Foundations of Computer Science (FOCS 2005), pp. 349-356. Washington, DC: IEEE Computer Society, 2005.

[Estrada 06] E. Estrada. "Spectral Scaling and Good Expansion Properties in Complex Networks." Europhysics Letters 73:4 (2006), 649-655.

[Faloutsos et al. 99] M. Faloutsos, P. Faloutsos, and C. Faloutsos. "On Power-Law Relationships of the Internet Topology." In SIGCOMM '99: Proceedings of the Conference on Applications, Technologies, Architectures, and Protocols for Computer Communication, pp. 251-262. New York: ACM Press, 1999.

[Feige and Ofek 05] U. Feige and E. Ofek. "Spectral Techniques Applied to Sparse Random Graphs." Random Structures and Algorithms 27:2 (2005), 251-275.

[Flaxman and Frieze 04] A. D. Flaxman and A. M. Frieze. "The Diameter of Randomly Perturbed Digraphs and Some Applications." In Approximation, Randomization and Combinatorial Optimization. Algorithms and Techniques: Proceedings 
of the 8th International Workshop on Randomization and Computation, RANDOM 2004, Cambridge, MA, USA, August 2004, Lecture Notes in Computer Science 3122, pp. 345-356, edited by K. Jansen, S. Khanna, J. D. P. Rolim, and D. Ron, Berlin: Springer, 2004.

[Flaxman et al. 04] A. Flaxman, A. M. Frieze, and J. Vera. "A Geometric Preferential Attachment Model of Networks." In Algorithms and Models for the Web-Graph: Third International Workshop, WAW 2004, Rome, Italy, October 16, 2004, Proceeedings, Lecture Notes in Computer Science 3243, pp. 44-55. Berlin: Springer, 2004 .

[Flaxman et al. 05] A. Flaxman, A. Frieze, and T. Fenner. "High Degree Vertices and Eigenvalues in the Preferential Attachment Graph." Internet Math. 2:1 (2005), $1-19$.

[Friedman 03] J. Friedman. "A Proof of Alon's Second Eigenvalue Conjecture." In Proceedings of the 35th Annual ACM Symposium on Theory of Computing, June 9-11, 2003, San Diego, CA, USA, pp. 720-724. New York: ACM Press, 2003.

[Friedman et al. 89] J. Friedman, J. Kahn, and E. Szemerédi. "On the Second Eigenvalue of Random Regular Graphs." In Proceedings of the Twenthy-First Annual ACM Symposium on Theory of Computing, Seattle, Washington, May 15-17, 1989, pp. 587-598. New York: ACM Press, 1989.

[Füredi and Komlós 81] Z. Füredi and J. Komlós. "The Eigenvalues of Random Symmetric Matrices." Combinatorica 1:3 (1981), 233-241.

[Janson et al. 00] S. Janson, T. Łuczak, and A. Rucinski. Random Graphs, WileyInterscience Series in Discrete Mathematics and Optimization. New York: WileyInterscience, 2000.

[Juhász 81] F. Juhász. "On the Spectrum of a Random Graph." In Algebraic Methods in Graph Theory, Colloquia Mathematica Societatis Janos Bolyai 25, pp. 313-316. Amsterdam: North-Holland, 1981.

[Kleinberg 00] J. Kleinberg. "The Small-World Phenomenon: An Algorithm Perspective." In Proceedings of the Thirty-Second Annual ACM Symposium on Theory of Computing, May 21-23, 2000, Portland, OR, USA, pp. 163-170. New York: ACM Press, 2000.

[Krivelevich et al. 06] M. Krivelevich, B. Sudakov, and P. Tetali. "On Smoothed Analysis in Dense Graphs and Formulas." Random Structures Algorithms 29:2 (2006), 180-193.

[Lawler and Sokal 88] G. F. Lawler and A. D. Sokal. "Bounds on the $L^{2}$ Spectrum for Markov Chains and Markov Processes: A Generalization of Cheeger's Inequality." Trans. Amer. Math. Soc. 309:2 (1988), 557-580.

[Mihail and Papadimitriou 02] M. Mihail and C. Papadimitriou. "On the Eigenvalue Power Law." In Randomization and Approximation Techniques in Computer Science, Lecture Notes in Computer Science 2483, pp. 254-262, Berlin: Springer, 2002 .

[Mihail et al. 03] M. Mihail, C. Papadimitriou, and A. Saberi. "On Certain Connectivity Properties of the Internet Topology." In Proceedings of the 44th Symposium 
on Foundations of Computer Science (FOCS 2003), p. 28. Washington, DC: IEEE Computer Society, 2003.

[Monasson 99] R. Monasson. "Diffusion, Localization and Dispersion Relations on 'Small-World' Lattices." The European Physical Journal B 12, 555-567 (1999).

[Sinclair and Jerrum 89] A. Sinclair and M. Jerrum. "Approximate Counting, Uniform Generation and Rapidly Mixing Markov Chains." Inform. and Comput. 82:1 (1989), 93-133.

[Spielman and Teng 01] D. Spielman and S.-H. Teng. "Smoothed Analysis of Algorithms: Why the Simplex Algorithm Usually Takes Polynomial Time." In Proceedings of the Thirty-Third Annual ACM Symposium on Theory of Computing, July 6-8, 2001, Heraklion, Crete, Greece, pp. 296-305, New York: ACM Press, 2001.

[Spielman and Teng 03] D. A. Spielman and S.-H. Teng. "Smoothed Analysis: Motivation and Discrete Models." In Algorithms and Data Structures, Lecture Notes in Computer Science 2748, pp. 256-270, Berlin: Springer, 2003.

[Watts and Strogatz 98] D. J. Watts and S. H. Strogatz. "Collective Dynamics of "Small-World Networks." Nature 292 (1998), 440-442.

[Wu 05] C. W. Wu. "Algebraic Connectivity of Directed Graphs." Linear Multilinear Algebra 53:3 (2005), 203-223.

Abraham D. Flaxman, Institute for Health Metrics and Evaluation, University of Washington, 2301 5th Ave., Suite 600, Seattle, WA 98121 (abie@u.washington.edu)

Received March 25, 2007; accepted July 31, 2007. 